\title{
The mystery of the disinterestedness regard Alopecia areata in Asians (especially Chinese men): A proposal of the use of a synthetic medicament plus a special herb from South America
}

\author{
Piotr Brzeziński ${ }^{1}$, Lorenzo Martini ${ }^{1,2}$, César Bimbi ${ }^{3}$
}

${ }^{1}$ University of Siena, Department of Pharmaceutical Biotechnologies, Via A. Moro 2, 53100 Siena, Italy, ${ }^{2}$ C.R.I.S.M.A. Inter

University Centre for Researched Advanced Medical Systems, ${ }^{3}$ Brazilian Society of Dermatology, Porto Alegre, Brazil

Corresponding author: Prof. Lorenzo Martini, E-mail: martini36@unisi.it

Sir,

Alopecia areata is believed to be an autoimmune condition with a worldwide occurrence. It usually presents as patchy, nonscarring hair loss. There is a paucity of clinical data in Asians [1].

Two hundred and nineteen new case referrals of alopecia areata were seen from May 1998 to April 1999. The incidence of alopecia areata was $3.8 \%$. There were 173 Chinese (79\%), 35 Indians (16\%), and 11 Malays $(5.0 \%)$. The male to female ratio was $1: 1.3$. The median age at presentation was 25.2 years. The majority of patients $(85.5 \%)$ had their first episode of alopecia areata before the age of 40 years. Of the patients with onset of alopecia areata before the age of 40 years, $36.5 \%$ presented with extensive alopecia, compared with $5.5 \%$ above the age of 40 years $(\mathrm{P}<0.05)$ [2-4].

In the majority of cases, hair falls out in small patches around the size of a quarter. For most people, the hair loss is nothing more than a few patches, though in some cases it can be more extreme.

Sometimes, it can lead to the complete loss of hair on the scalp (alopecia totalis) or, in extreme cases, the entire body (alopecia universalis).

One in five people with alopecia areata also has a family member who has experienced the condition $[4,5]$.

- Alopecia areata often develops suddenly, over the course of just a few days.
- There is little scientific evidence that alopecia areata is caused by stress.

- People with alopecia areata who have only a few patches of hair loss often experience a spontaneous, full recovery, without the need for treatment.

- There is no cure for alopecia areata.

Share on Pinterest Alopecia areata is a common autoimmune disorder that commonly results in unpredictable hair loss.

There is currently no cure for alopecia areata, although there are some forms of treatment that can be suggested by doctors to help hair re-grow more quickly.

The most common form of alopecia areata treatment is the use of corticosteroids, powerful anti-inflammatory drugs that can suppress the immune system. These are mostly commonly administered through local injections, topical ointment application, or orally $[5,6]$.

Other medications that can be prescribed that either promote hair growth or affect the immune system include Minoxidil, Anthralin, SADBE, and DPCP. Although some of these may help with the re-growth of hair, they cannot prevent the formation of new bald patches [7].

The use of photochemotherapy is supported by some studies and presents a potential alternative for patients unable or unwilling to use systemic or invasive therapies.

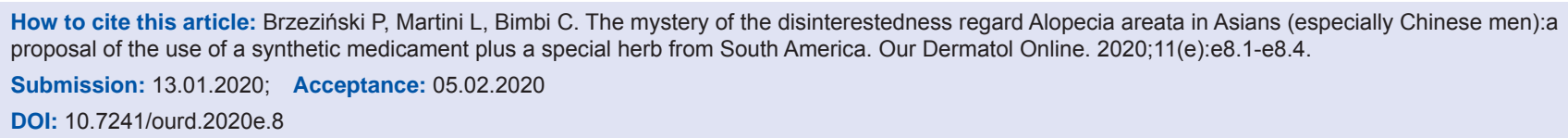


In addition to its aesthetic aspect, hair affords a degree of protection against the elements. People with alopecia areata who miss the protective qualities of hair may wish to:

- Wear sunscreen if exposed to the sun.

- Wear wraparound glasses to protect the eyes from the sun and debris which the eyebrows and eyelashes would normally defend against.

- Use headwear such as hats, wigs, and scarves to protect the head from the sun or keep it warm.

- Use ointment inside the nose to keep membranes moist and to protect against organisms that are normally trapped by nostril hair.

Alopecia areata does not directly make people sick, nor is it contagious. It can, however, be difficult to adapt to emotionally. For many people, alopecia areata is a traumatic disease that warrants treatment addressing the emotional aspect of hair loss, as well as the hair loss itself $[7,8]$.

Support groups and counseling are available for people to share their thoughts and feelings, and to discuss common psychological reactions to the condition.

Alopecia areata has been compared by some to vitiligo, an autoimmune skin disease where the body attacks melanin-producing cells, leading to white patches. Research suggests that these two conditions may share a similar pathogenesis, with similar types of immune cells and cytokines driving the diseases and common genetic risk factors.

As such, any new developments in the treatment or prevention of either disease may have consequences for the other.

Preliminary research in animals has found that quercetin, a naturally occurring bioflavonoid found in fruits and vegetables, can protect against the development of alopecia areata and effectively treat existing hair loss.

Further research is needed, including human clinical trials, before quercetin can be considered a treatment for alopecia areata.

Inizio modulo

The condition occurs when white blood cells attack the cells in hair follicles, causing them to shrink and dramatically slow down hair production. It is unknown precisely what causes the body's immune system to target hair follicles in this way.

While scientists are unsure why these changes occur, it seems that genetics are involved as alopecia areata is more likely to occur in a person who has a close family member with the disease. One in five people with the disease has a family member who has also developed alopecia areata.

Other research has found that many people with a family history of alopecia areata also have a personal or family history of other autoimmune disorders, such as atopy, a disorder characterized by a tendency to be hyperallergic, thyroiditis, and vitiligo.

Despite what many people think, there is very little scientific evidence to support the view that alopecia areata is caused by stress. Extreme cases of stress could potentially trigger the condition, but most recent research points toward a genetic cause [6-8].

Powered by Rubicon Project

As conventional treatments for alopecia are extremely limited, studies that support natural treatments for alopecia are even thinner on the ground.

There are some people that recommend rubbing onion or garlic juice, cooled green tea, almond oil, rosemary oil, honey, or coconut milk into the scalp. While none of these are likely to cause harm, their effectiveness is also not supported by research.

Some people turn to alternative treatment methods such as acupuncture and aromatherapy, although there is little, if any, evidence to support these treatments.

The most prominent symptom of alopecia areata is patchy hair loss. Coin-sized patches of hair begin to fall out, mainly from the scalp. Any site of hair growth may be affected, though, including the beard and eyelashes.

The loss of hair can be sudden, developing in just a few days or over a period of a few weeks. There may be itching or burning in the area before hair loss. The hair follicles are not destroyed and so hair can re-grow if the inflammation of the follicles subsides. People who experience just a few patches of hair loss often have a spontaneous, full recovery without any form of treatment.

About 30 percent of individuals who develop alopecia areata find that their condition either becomes more 
extensive or becomes a continuous cycle of hair loss and regrowth.

About half of patients recover from alopecia areata within 1 year, but many will experience more than one episode. Around 10 percent of people will go on to develop alopecia totalis or alopecia universalis.

Alopecia areata can also affect the fingernails and toenails, and sometimes these changes are the first sign that the condition is developing. There are a number of small changes that can occur to nails:

Share on Pinterest Alopecia affects both men and women equally.

- pinpoint dents appear; white spots and lines appear; nails become rough; nails lose their shine; nails become thin and split.

Doctors are usually able to diagnose alopecia areata fairly easily by examining symptoms. They might look at the degree of hair loss and examine hairs from affected areas under a microscope.

If, after an initial clinical examination, the doctor is not able to make a diagnosis, they can perform a skin biopsy. If they need to rule out other autoimmune diseases, they might perform a blood test.

As the symptoms of alopecia areata are so distinctive, making a diagnosis is usually quick and straightforward.

The AA of this paper has decided to use corticosteroids for topical uses together with the aqueous extract of Impatiens balsamina.

The flowers of garden balsam (Begliuomini in Italian) contain the same dyeing agent as henna, lawsone or 2-hydroxy-1,4-naphthaquinone, which explains the matching uses. Also present is 2-methoxy-1,4naphthaquinone (lawsone-methylether) which possesses fungicidal properties. The pigments in the flowers have been investigated extensively, and include leucoanthocyanins, anthocyanins and flavonols. The seeds contain 18-27\% of a greenish, viscous oil, largely consisting of parinaric acid (29\%) and linolenic acid (30\%). The seeds contain about $16 \%$ protein and no starch.

Leaves and sometimes roots are used in poultices for wounds, skin diseases, manifold pustules, felons.

They have used the aqueous extract of leaves and flowers of $\mathrm{B}$. impatiens, to mix together with triamcinolone acetonide $(40 \mathrm{mg} / \mathrm{ml})(50: 50)$ and recruited an Asian man (32 y. old) with an impressive and extended alopecia areata (causes surely were malnutrition, paucity of Zinc ion and vitamins and especially emotive stress).

In the formula pilocarpine (1\%) and nicotinic acid (2\%) to let the regrowth of hair were included.

The AA prayed the volunteer to apply with a wool swab twice a day (morning and evening) the aqueous lotion.

Two applications pro day for two entire weeks have been sufficient to observe a neat decrease of the ampleness and contour of the white spot and a valuable regrowth of hair in the white spots.

Moreover, thanks to the lawsone of the flowers of the Impatiens balsamina, white spots began flesh colored.

The AA are very satisfied for the success, even because there is really a scarcity of researches and studies on alopecia areata in Asians.

\section{AKNOWLEDGMENTS}

The possibility to have the titrated triamcinolone acetonide in deionized water has been possible owing to the intervention of Dr. Domitilla Ordini, one of the few Dermatologists in our town where we can boast the first important University of all Italy.

\section{Consent}

The examination of the patient was conducted according to the Declaration of Helsinki principles.

The authors certify that they have obtained all appropriate patient consent forms. In the form the patient(s) has/have given his/her/their consent for his/ her/their images and other clinical information to be reported in the journal. The patients understand that their names and initials will not be published and due efforts will be made to conceal their identity, but anonymity cannot be guaranteed.

\section{REFERENCES}

1. Tan E, Tay YK, Goh CL, Chin Giam Y. The pattern and profile of alopecia areata in Singapore-a study of 219 Asians. Int J Dermatol. 2002;41:748-53.

2. Biran R, Zlotogorski A, Ramot Y. The genetics of alopecia areata: new approaches, new findings, new treatments. J Dermatol Sci. 


\section{www.odermatol.com}

2015;78:11-22.

3. Gilhar A, Etzioni A, Paus R. Alopecia areata. N Engl J Med. 2012;19;366:1515-25.

4. Jabbari A, Petukhova L, Cabral RM, Clynes R, Christiano AM. Genetic basis of alopecia areata: a roadmap for translational research. Dermatol Clin. 2013;31:109-11.

5. Korta DZ, Christiano AM, Bergfeld W, Duvic M, Ellison A, Fu J, et al. Alopecia areata is a medical disease. J Am Acad Dermatol. 2018:78:832-4.

6. Petukhova L, Christiano AM. The genetic architecture of alopecia areata. J Investig Dermatol Symp Proc. 2013;16:16-22.

7. Pratt CH, King LE Jr, Messenger AG, Christiano AM, Sundberg JP.
Alopecia areata. Nat Rev Dis Primers. 2017;3:17011.

8. Strazzulla LC, Wang EHC, Avila L, Lo Sicco K, Brinster N, Christiano AM, et al. Alopecia areata: Disease characteristics, clinical evaluation, and new perspectives on pathogenesis. J Am Acad Dermatol. 2018;78:1-12.

Copyright by Piotr Brzeziński, et al. This is an open-access article distributed under the terms of the Creative Commons Attribution License, which permits unrestricted use, distribution, and reproduction in any medium, provided the original author and source are credited.

Source of Support: Nil, Conflict of Interest: None declared. 